Research Article

\title{
Neural Network Optimization Method and Its Application in Information Processing
}

\author{
Pin Wang, ${ }^{1}$ Peng Wang $\mathbb{D}^{2},{ }^{2}$ and En Fan $^{3}$ \\ ${ }^{1}$ School of Mechanical and Electrical Engineering, Shenzhen Polytechnic, Shenzhen 518055, Guangdong, China \\ ${ }^{2}$ Garden Center, South China Botanical Garden, Chinese Academy of Sciences, Guangzhou 510650, Guangdong, China \\ ${ }^{3}$ Department of Computer Science and Engineering, Shaoxing University, Shaoxing 312000, Zhejiang, China
}

Correspondence should be addressed to Peng Wang; sdhzdtwp@126.com

Received 3 December 2020; Revised 31 December 2020; Accepted 22 January 2021; Published 8 February 2021

Academic Editor: Sang-Bing Tsai

Copyright (C) 2021 Pin Wang et al. This is an open access article distributed under the Creative Commons Attribution License, which permits unrestricted use, distribution, and reproduction in any medium, provided the original work is properly cited.

Neural network theory is the basis of massive information parallel processing and large-scale parallel computing. Neural network is not only a highly nonlinear dynamic system but also an adaptive organization system, which can be used to describe the intelligent behavior of cognition, decision-making, and control. The purpose of this paper is to explore the optimization method of neural network and its application in information processing. This paper uses the characteristic of SOM feature map neural network to preserve the topological order to estimate the direction of arrival of the array signal. For the estimation of the direction of arrival of single-source signals in array signal processing, this paper establishes a uniform linear array and arbitrary array models based on the distance difference vector to detect DOA. The relationship between the DDOA vector and the direction of arrival angle is regarded as a mapping from the DDOA space to the AOA space. For this mapping, through derivation and analysis, it is found that there is a similar topological distribution between the two variables of the sampled signal. In this paper, the network is trained by uniformly distributed simulated source signals, and then the trained network is used to perform AOA estimation effect tests on simulated noiseless signals, simulated Gaussian noise signals, and measured signals of sound sources in the lake. Neural network and multisignal classification algorithms are compared. This paper proposes a DOA estimation method using two-layer SOM neural network and theoretically verifies the reliability of the method. Experimental research shows that when the signal-to-noise ratio drops from $20 \mathrm{~dB}$ to $1 \mathrm{~dB}$ in the experiment with Gaussian noise, the absolute error of the AOA prediction is small and the fluctuation is not large, indicating that the prediction effect of the SOM network optimization method established in this paper does not vary. The signal-to-noise ratio drops and decreases, and it has a strong ability to adapt to noise.

\section{Introduction}

In the information society, the increase in information generation is getting bigger [1]. To make information available in a timely manner to serve the development of the national economy, science and technology, and defense industry, it is necessary to collect, process, transmit, store, and make decisions on information data. Theoretical innovation and implementation are carried out to meet the needs of the social development situation. Therefore, neural networks have extremely extensive research significance and application value in information science fields such as communications, radar, sonar, electronic measuring instruments, biomedical engineering, vibration engineering, seismic prospecting, and image processing. This article focuses on the study of neural network optimization methods and their applications in intelligent information processing.

Based on the research of neural network optimization method and its information processing, many foreign scholars have studied it and achieved good results. For example, Al Mamun MA has developed a new method of image restoration using neural network technology, which overcomes to a certain extent the above shortcomings of traditional methods. In addition, neural networks have also been widely used in image edge detection, image segmentation, and image compression [2]. Hamza MF proposed a $\mathrm{BP}$ algorithm to train RBF weights. The BP algorithm with additional momentum factor can improve the training 
coefficient of the network and avoid the occurrence of oscillations, which improves the training rate of the network [3]. Tom B proposed an RBF-PLSR model based on genetic clustering. This model uses the clustering analysis of genetic algorithm to determine the number of hidden layer nodes and the center of hidden nodes in the RBF network, and the PLSR method is used to determine the network's right to connect $[4,5]$.

In my country, an adaptive linear component model is proposed. They also made Adaline into hardware and successfully applied it to offset the echo and noise in communications. Quan proposed the error back-propagation algorithm, the BP algorithm, which in principle solves the problem of the multilayer neural network training method, which makes the neural network have strong computing power and greatly increases the vitality of the artificial neural network [6]. Cheng uses mathematical theory to prove the fundamental limitation of single-layer perceptron in computing. However, for multilayer neural networks with hidden layers, an effective learning algorithm has not yet been found [7].

In this paper, the problem of single-signal source azimuth detection under uniform linear sensor array and arbitrary array is studied, and the direction-of-arrival detection array model is established, respectively. In the case of a uniform linear array, this paper establishes a twolayer SOM neural network. First, explain the theoretical basis of this neural network, that is, the homotopological structure between the input vector and the output result. For this reason, we separately analyzed the topological structure of the DDOA vector and the predicted value of the AOA in the case of a uniform linear array. Through derivation and simulation data, we can see that the two do have similar topological structures, which led us to establish the SOM neural network system. It can be applied to AOA prediction problems based on DDOA. Finally, simulation experiments and lake water experiments verify the practical feasibility of this method.

\section{Neural Network Optimization Method and Its Research in Information Processing}

2.1. Array Optimization and Orientation Based on DDOA and SOM Neural Network. Signal and information processing mainly includes three main processes: information acquisition, information processing, and information transmission $[8,9]$. The array signal processing can be regarded as an important branch of modern data signal processing. Its main research object is the signal transmitted in the form of spatial transmission wave. It receives the wave signal through a sensor array with a certain spatial distribution and performs information on the received signal extract. This paper mainly studies the algorithm of the sensor array to detect the sound wave's azimuth, namely, the direction of arrival (DOA).

2.1.1. Array Signal Model. Array signal processing is often based on a strict mathematical theoretical model based on a series of assumptions about the observed signal. The objects explored in this article are all two-dimensional spatial signal problems. These assumptions stem from the abstraction and generalization of the observed signal and noise.

(1) Narrowband signal: when the bandwidth of the spatial source signal is much smaller than its center frequency, we call this spatial source signal a narrowband signal; that is, the general requirement is met.

$$
\frac{W_{B}}{f_{o}}<\frac{1}{10},
$$

where $W_{B}$ is the signal bandwidth and $f_{o}$ is the signal center frequency. A single-frequency signal with a center frequency of $f_{o}$ can be used to simulate a narrowband signal. The sine signal as we know it is a typical narrowband signal. The analog signals used in this article are all single-frequency sine signals.

(2) Array signal processing model: suppose that there is a sensor array in the plane, in which $M$ sensor array elements with arbitrary directivity are arranged, and $K$ narrowband plane waves are distributed in this plane. The center frequencies of these plane waves are all $w_{o}$ and the wavelength is $\lambda$, and suppose that $M>K$ (that is, the number of array elements is greater than the number of incident signals). The signal output received by the $k$-th element at time $t$ is the sum of K plane waves; namely,

$$
x_{k}(t)=\sum_{t=1}^{K} a_{k}\left(\theta_{i}\right) s_{i}\left(t-\tau_{k}\left(\theta_{i}\right)\right),
$$

where $a_{k}\left(\theta_{i}\right)$ is the sound pressure response coefficient of element $k$ to source $i, s_{i}\left(t-\tau_{k}\left(\theta_{i}\right)\right)$ is the signal wavefront of source $i$, and $\tau_{k}\left(\theta_{i}\right)$ is the relative value of element $k$ to the reference element time delay. According to the assumption of narrowband waves, the time delay only affects the wavefront by the phase change, so

$$
s_{i}\left(t-\tau_{k}\left(\theta_{i}\right)\right) \approx s_{i}(t) e^{-j w_{0} \tau_{k}\left(\theta_{i}\right)} .
$$

Therefore, formula (2) can be rewritten as

$$
x_{k}(t)=\sum_{i=1}^{K} a_{k}\left(\theta_{i}\right) s_{i}(t) e^{-j w_{0} \tau_{k}\left(\theta_{i}\right)} .
$$

Write the output of $M$ sensors in vector form; the model becomes

$$
x(t)=\sum_{i=1}^{K} a\left(\theta_{i}\right) s_{i}(t) .
$$

Among them,

$$
\begin{aligned}
a\left(\theta_{i}\right)= & {\left[a_{1}\left(\theta_{i}\right) e^{-j w_{0} \tau_{1}\left(\theta_{i}\right)}, a_{2}\left(\theta_{i}\right) e^{-j w_{0} \tau_{2}\left(\theta_{i}\right)},\right.} \\
& \left.\ldots, a_{M}\left(\theta_{i}\right) e^{-j w_{0} \tau_{3}\left(\theta_{i}\right)}\right]
\end{aligned}
$$

It is called the direction vector of the incoming wave direction 0 . 
Let $\quad A(\theta)=\left[a\left(\theta_{1}\right), a\left(\theta_{1}\right), \ldots, a\left(\theta_{1}\right)\right], s(t)=\left[s_{1}\right.$ $\left.(t), s_{1}(t), \ldots, s_{1}(t)\right]^{T}$. The other measurement noise is $n(t)$; then the above array model can be expressed as

$$
x(t)=A(\theta) s(t)+n(t) .
$$

Among them, $x(t), n(t) \in C^{K}$, and $A(\theta) \in C^{M \times K}$ is the direction matrix of the array model.

\subsubsection{Subspace Decomposition Based on Eigendecomposition} of Array Covariance Matrix. The DOA estimation problem is the estimation of the direction of arrival angle and the parameter $\theta_{i}(i=1,2, \ldots, K)$ in natural space, which requires the covariance information between the different elements of the array for analysis. For this, first calculate the spatial covariance matrix output by the array:

$$
R=E\left[x(t) x^{H}(t)\right]
$$

where $E\{$.$\} represents statistical expectation; let$

$$
\begin{aligned}
& E\left[s(t) s^{H}(t)\right]=R_{s}, \\
& E\left[n(t) n^{H}(t)\right]=\sigma^{2} I .
\end{aligned}
$$

It is the covariance matrix of noise. It is assumed that the noise received by all elements has a common variance, and $\sigma^{2}$ is also the noise power. From equations (9) and (10), we can get

$$
R=A R_{s} A^{H}+\sigma^{2} I .
$$

It can be proved that $R$ is a nonsingular matrix and a positive definite Hermitian square matrix; that is, $R^{H}=R$. Therefore, the singular value decomposition of $R$ can be performed to achieve diagonalization, and the eigendecomposition can be written as follows:

$$
R=U \Lambda U^{H},
$$

where $U$ is the transformation unitary matrix, so that matrix $R$ is diagonalized into a real-valued matrix $\Lambda=\operatorname{diag}\left(\lambda_{1}, \lambda_{2}, \ldots, \lambda_{M}\right)$, and the eigenvalues are ordered as follows:

$$
\lambda_{1} \geq \lambda_{2} \geq \cdots \geq \lambda_{M}>0 .
$$

From equation (13), it can be seen that any vector orthogonal to $A$ is an eigenvector of matrix $R$ belonging to the eigenvalue $\sigma^{2}$.

2.1.3. RBF Neural Network Estimates the Direction of Arrival. $\mathrm{RBF}$ neural network is a method that can perform curve fitting or interpolation in high-dimensional space. If the relationship between the input space and the output space is regarded as a mapping, this mapping can be regarded as defined in the high-dimensional space. A hypersurface of the input data and a designed RBF neural network are equivalent to the height fitting of this hypersurface. It establishes an approximate hypersurface by interpolating the input data points $[10,11]$.

The sensor array is equivalent to a mapping from the DOA space $\left(\left\{\theta=\left[\theta_{1}, \theta_{2}, \ldots, \theta_{K}\right]\right\}\right)$ to the sensor array output space $\left(\left\{s=\left[s_{1}, s_{2}, \ldots, s_{M}\right]\right\}\right), \quad$ a mapping $G: R^{K} \longrightarrow C^{M}$ :

$$
s_{m}=\sum_{k=1}^{K} a_{k} e^{j\left(\left(m \omega_{0} d \sin \theta_{k} / c\right)+a_{k}\right)}, \quad m=1,2, \ldots, M,
$$

where $K$ is the number of source signals, $M$ is the number of elements of the uniform linear array, $a_{k}$ is the complex amplitude of the $k$-th signal, $\alpha$ is the initial phase, $\omega_{0}$ is the signal center frequency, $d$ is the element spacing, and $c$ is the propagation speed of the source signal $[12,13]$.

When the number of information sources has been estimated as $K$, the function of the neural network on this problem is equivalent to the inverse problem of the above mapping, that is, the inverse mapping $\mathrm{F}: C^{M} \longrightarrow R^{K}$. To obtain this mapping, it is necessary to establish a neural network structure in which the preprocessed data based on the incident signal is used as the network input, and the corresponding DOA is used as the network output after the hidden layer activation function is applied. The whole process is a targeted training process, and the process of fitting the mapping with the RBF neural network is equivalent to an interpolation process.

\subsection{Estimation of Direction of Arrival of Uniform Linear Array SOM Neural Network}

2.2.1. Kohonen Self-Organizing Neural Network. A SOM neural network consists of two layers: the input layer and the competition layer (also called the output layer). The number of nodes in the input layer is equal to the dimension of the input vector, and the neurons in the competing layer are usually arranged in a rectangle or hexagon on a two-dimensional plane. The output node $j$ and the input node are connected by weights:

$$
\omega_{j}=\left[\omega_{j 1}, \omega_{j 2}, \ldots, \omega_{j m}\right]^{T} .
$$

The training steps of the Kohonen SOM neural network used in this article are as follows: the first step is network initialization $[14,15]$. Normalize the input vector $x$ to $\hat{x}$ such that $|(|\hat{\mathrm{x}}|)|=1$ :

$$
\widehat{x}=\frac{x}{\|x\|}=\frac{x}{\left[\sum_{i=1}^{m}\left(x_{1}\right)^{2}\right]^{1 / 2}},
$$

where $x=\left[x_{1}, x_{2}, \ldots, x_{m}\right]^{T}$ is the training sample vector of the network. Initialize the network weight $w_{j}$ $(j=1,2, \ldots, K)$ to be the same as the partially normalized input vector é.

The second step is to calculate the Euclidean distance between the input vector $\hat{x}$ and the corresponding weight vector $\omega_{j}$ of each competing layer neuron to obtain the 
winning neuron $\omega_{c}[16,17]$. The selection principle of the winning neuron is as follows:

$$
\left\|\widehat{x}-t \omega_{c}\right\|=\min _{j}\left\|\widehat{x}-t \omega_{j}\right\| .
$$

The third step is to adjust the weight of the winning neuron $\omega_{c}$ and its neighborhood $\omega_{j}$. The adjustment method is as follows:

$$
\omega_{j i}(t+1)=\omega_{j i}(t)+\eta(t)\left[\widehat{x}_{i}-\omega_{j i}(t)\right], \quad j \in U_{c}(t) .
$$

Among them, $\eta(t)$ is the learning rate function, which decreases with the number of iteration steps $t[18,19]$. The function $U_{c}(t)$ is the neighborhood function; here is the Gaussian function:

$$
U_{c}(t)=\exp \left(\frac{\left\|r_{j}-r_{c}\right\|^{2}}{2 \sigma^{2}}\right) \eta(t),
$$

where $r$ is the position of the neurons in the competition layer on a two-dimensional plane and $\sigma$ is the smoothing factor, which is a normal number.

2.2.2. DOA Estimation Model Based on SOM Neural Network. Build a two-layer SOM neural network. The first layer of SOM neural network is the sorting layer, which maps the input training data into a two-dimensional space. According to the activation of neuron nodes on the first twodimensional grid, the output of the corresponding neuron node in the second grid is defined by the following rules:

(1) If the neuron node $j$ is activated by only one training sample vector and the signal position corresponding to this sample is $\left(x_{k_{i}}, y_{k_{i}}\right)\left(i=1,2, \ldots, n_{j}\right)$, then the output of the corresponding node of the second layer of grid is the direction angle of this signal $[20,21]$, namely,

$$
\theta_{j}=\arctan \frac{y_{i}}{x_{i}-M(r / 2)}
$$

(2) If the neuron node $j$ is activated by more than one training sample vector, that is, $n_{j}>1$, and the signal positions corresponding to these samples are $\left(x_{k_{i}}, y_{k_{i}}\right)\left(i=1,2, \ldots, n_{j}\right)$, then the output of the corresponding node of the second layer of grid is the average value of the direction angle of these signals $[22,23]$, namely,

$$
\theta_{j}=\frac{1}{n_{j}} \sum_{i=1}^{n_{j}} \arctan \frac{y_{k_{i}}}{x_{k_{i}}-M(r / 2)} .
$$

(3) If the neuron node $j$ has never been activated by any training sample vector, the corresponding output neuron node is regarded as an invalid node. When this node is activated by a new input vector, the output value is defined as the output direction angle of the valid node closest to this node.
2.2.3. Method Reliability Analysis. The establishment process of the two-layer SOM neural network we proposed above shows that the topological order of AOA is similar to the topological distribution of DDOA vectors. In other words, when the Euclidean distance between two DDOA vectors is small, the Euclidean distance of the corresponding AOA value must also be small. This is the theoretical basis for our proposed method, and we will conduct a detailed analysis on this nature.

Suppose that the DDOA vectors of two adjacent source signals are $d$ and $d_{1}=d+\Delta d$, and the corresponding AOAs are $\theta$ and $\theta_{1}=\theta+\Delta \theta$, respectively. The DDOA increment and AOA increment are

$$
\begin{aligned}
& \Delta d=\left[\Delta d_{0,1}, \Delta d_{1,2}, \ldots, \Delta d_{M-1, M}\right]^{T}, \\
& \Delta \theta=\theta_{1}-\theta,
\end{aligned}
$$

where $\Delta d_{i, j+1}=d_{i, j+1}^{1}-d_{i, j+1}$; obviously the function $d_{i, j+1}$ at the point $(x, y) \in R^{2}$ is differentiable, which shows that the DDOA vector $d$ and the AOA value $\theta$ have a consistent trend $[24,25]$. In other words, when the DDOA vectors of two source signals are similar, their arrival direction angles AOA must also be similar. Therefore, the topological orders and distributions of DDOA vector and AOA are basically the same.

2.3. Genetic Clustering Method. In cluster analysis, the $\mathrm{K}$-means clustering method is a clustering method that is often used. Generally, when determining the structure of the RBF network, this method is used to determine the number of hidden layer nodes of the network and the center of the node.

\subsubsection{Chromosome Coding and Population Initialization.} In order to accelerate the speed of convergence, we use real number coding [26]. For samples with $m$-dimensional dimensions, if the number of classes to be classified is $n$, the centers of $n$ classes are encoded, and the dimensions of each center are $m$-dimensional; then the length of the chromosome is $n \times m$. In this way, a chromosome represents a complete classification strategy. Initialize the preset number of chromosomes to get the initial population.

\subsubsection{Determination of Fitness Function and Selection of} Fitness. For each chromosome, according to the classification information carried on it, according to the idea of distance classification, the classification of each sample in the original data can be determined, and the distance between the sample and its category center (here is Euclidean distance) can also be determined [27, 28]. After determining the classification of the sample, the sum of the distances within the class can be calculated:

$$
F=\sum_{i=1}^{k} \sum_{j=1}^{n_{i}}\left\|x_{j}-C_{i}\right\| .
$$


At the same time, the sum of the distances between classes can also be found:

$$
Q=\frac{1}{2} \sum_{i=1}^{k} \sum_{j=1, j \neq 1}^{k}\left\|C_{i}-C_{j}\right\|,
$$

where $F$ is the sum of distances within classes, $Q$ is the sum of distances between classes, $k$ is the number of classes in the classification, $n_{i}$ is the number of samples belonging to the $i$ th class, $x_{j}$ is the $j$-th sample of the $i$-th class, and $C_{i}$ is the center of the $i$-th class.

\section{Neural Network Optimization Method and Its Experimental Research in Information Processing}

3.1. Underwater Experimental Research in the Lake. The underwater experiment is carried out in the lake. The average depth of the lake water is 50 meters to 60 meters. The area of open water is more than 300 meters $* 1200$ meters, and the water body is relatively stable and suitable for DOA estimation experiments. The experimental equipment used this time is a uniform linear array composed of 4 acoustic pressure hydrophones with an array spacing of 0.472 meters.

\subsection{Experimental Methods and Data Collection}

3.2.1. No Noise. In order to verify the effectiveness of the two-layer SOM neural network established in this paper for arbitrary array conditions, we conducted a simulation experiment of detecting the direction of acoustic signals with arbitrary sensor arrays underwater. Assuming that the sensor array contains 4 sensors, the frequency of a single sound source signal is $f=2 \mathrm{kHz}$, the propagation speed of the sound signal in water is $c=1500 \mathrm{~m} / \mathrm{s}$, and the distance between two adjacent sensors is $\Delta_{i}=0.375$, which is the wavelength half. The positions of the four sensor array elements are $\left(x_{1}=0 . y_{1}=0\right),\left(x_{2}=0.3, y_{2}=0.225\right),\left(x_{3}=0.5\right.$, $\left.y_{3}=-0.0922\right)$, and $\left(x_{4}=0.6, y_{4}=0.2692\right)$. In order to obtain the training vector, we uniformly collect $60 \times 30$ points from the rectangular area $[-20,20] \times[0,20] \in R^{2}$ as the emission positions of 1800 simulated sound source signals, which can calculate 1800 DDOA vectors $r$, and input them into the network as training vectors of the network.

Calculate the value of $R_{\max }(x, y)$ :

$$
\begin{aligned}
R_{\max }(x, y)= & \max R(x, y, \Delta x, \Delta y), \quad(x, y) \in[-100,100] \\
& \times[-100,100] .
\end{aligned}
$$

Except for the few points near the origin $(0,0)$, the function $R_{\max }(x, y)$ at most of the remaining points has a common upper bound, which belongs to the second case.

3.2.2. Noise. In practice, the signal data collected by the sensor array is often noisy, and the energy of noise is generally large. The signal-to-noise ratio between signal and noise often reaches very low values, even below $0 \mathrm{~dB}$; that is, the signal is overwhelmed by environmental noise that is much stronger than its strength. When the signal-to-noise ratio is particularly small, people usually perform a denoising filtering process artificially in advance to make the filtered signal-to-noise ratio at least above $0 \mathrm{~dB}$. Therefore, a good model that can be applied to practice must be applicable to noisy environments.

\subsection{Performing Genetic Clustering on Standardized Training} Sample Data. The number of preselected clustering categories is in the interval between $1 / 7$ and $1 / 4$ of the total number of samples (in order to facilitate the training of the network, too few or too many categories will result in poor training effects), take the population number as 30 , the crossover rate is $75 \%$, and the mutation rate is $5 \%$. The fitness function is selected so that the ratio of the interclass distance to the intraclass distance increases with the increase of the fitness function, and a convergent solution can be obtained in about 50 generations. In the interval class, the number of classes is changed one by one until the fitness function is minimized. The number of categories at this time is the number of hidden nodes in the RBF network, and the center of each category is the center of the node.

\section{Neural Network Optimization Method and Its Experimental Research Analysis in Information Processing}

4.1. Noise-Free Simulation Experiment. To test the performance of the network, we select six sets of source signals with different distances from the origin, that is, six sets of points as the test. The distances to the origin of the coordinates are 8 meters, 16 meters, 20 meters, 30 meters, 50 meters, and 100 meters. Each group contains 21 simulated signals with different AOA values. Calculate the DDOA vectors corresponding to these simulated signal emission points, and then input these vectors as test vectors into the trained two-layer SOM neural network. The output of the network is the corresponding AOA predicted value. The experimental results are shown in Figure 1.

The absolute error value of the AOA prediction result is shown in Figure 1. It can be seen that not only can the SOM network trained with the near-field simulation signal (the signal position is within the area $[0,21] \times[0,21])$ perform the training in the near-field $(4 \mathrm{~m}, 8 \mathrm{~m}$, and $12 \mathrm{~m}$ ) but also the AOA prediction effect of the test signal is good. Except for individual points, the AOA prediction accuracy of the test signal $(16 \mathrm{~m}, 32 \mathrm{~m}$, and $64 \mathrm{~m})$ in the far field is also very high, and the error is basically controlled in the interval $\left[-0.4^{0}, 0.4^{0}\right]$, the error is smaller than the near field, the effect is better, and the error fluctuation is smaller.

To illustrate the effectiveness and scalability of this method in predicting AOA, we set up an RBF neural network for comparison. The RBF neural network established here uses the DDOA vector of the same simulation signal (within the area $[0,20][0,20]$ ) as the input vector of the 


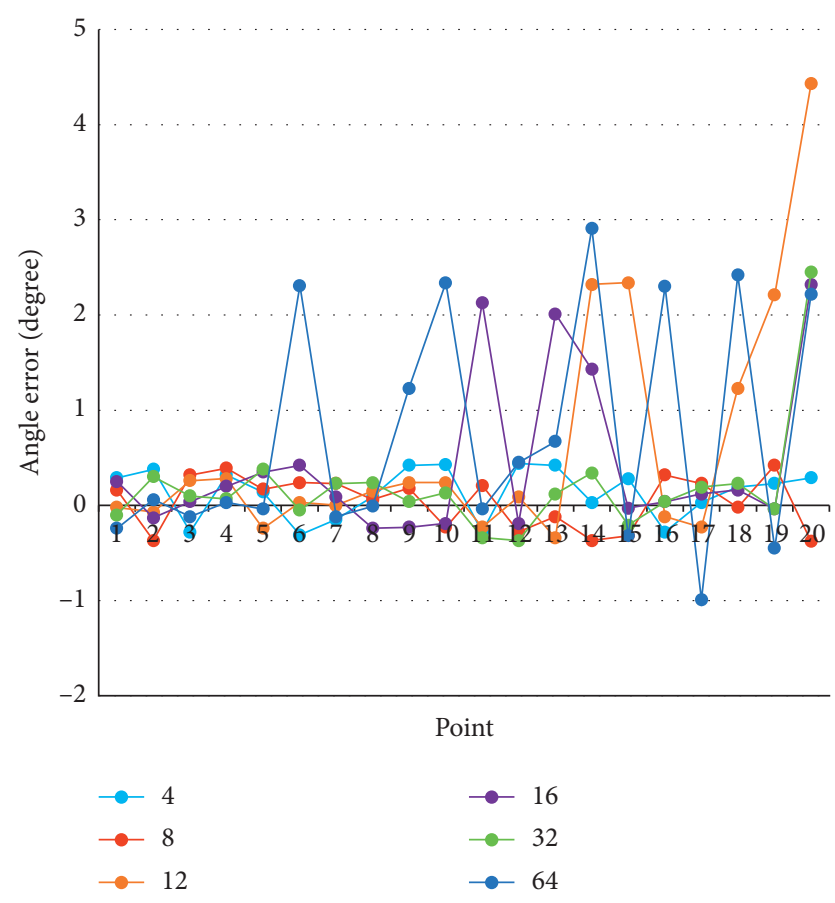

FIGURE 1: Absolute error of AOA predicted value of source signal at different distances using SOM neural network.

network training and the corresponding AOA value as the target output of the network training.

As shown in Table 1 and Figure 2, the average of the absolute value of the AOA prediction error of the noise-free signal in the simulation experiment is approximately $0.1^{\circ}$ to $0.4^{\circ}$, the minimum is $0.122^{\circ}$, and the maximum is only $0.242^{\circ}$, and most of the test signals (accounting for the absolute value of the prediction error of the number of test signals $(70 \% \sim 80 \%))$ are less than $0.1^{\circ}$.

To illustrate the effectiveness and scalability of this method in predicting AOA, we set up an RBF neural network for comparison. The RBF neural network established here uses the DDOA vector of the same simulation signal (within the area $[0,20][0,20]$ ) as the input vector for network training, and the corresponding AOA value is used as the target output of the network training. Experimental results are shown in Table 2 .

As shown in Figure 3, the two networks both use the same 20 simulated signals as test signals. The transmission positions of the tested signals are evenly distributed between 2 meters and 40 meters from the origin, including the training area, that is, within 20 meters. It can be seen from Figure 4 that the prediction effect of the RBF neural network in the training area is similar to that of the SOM neural network, but the prediction effect outside the training area is poor, while the SOM neural network shows strong adaptability to distance changes. This shows that the RBF neural network will be affected by the distance factor, because its training principle is to use the idea of interpolation to fit the mapping relationship, which makes the error larger when the test data exceeds the training range.
TABLE 1: SOM neural network prediction results of noise-free signal AOA.

\begin{tabular}{lcccccc}
\hline Distance & 4 & 8 & 12 & 16 & 32 & 64 \\
\hline Average error & 0.215 & 0.124 & 0.147 & 0.105 & 0.109 & 0.152 \\
$\operatorname{Pr}\left(\right.$ err $\left.<0.3^{\circ}\right)$ & 0.763 & 0.862 & 0.901 & 0.986 & 0.853 & 0.901 \\
$\operatorname{Pr}\left(\right.$ err $\left.<0.2^{\circ}\right)$ & 0.782 & 0.816 & 0.792 & 0.827 & 0.879 & 0.815 \\
\hline
\end{tabular}

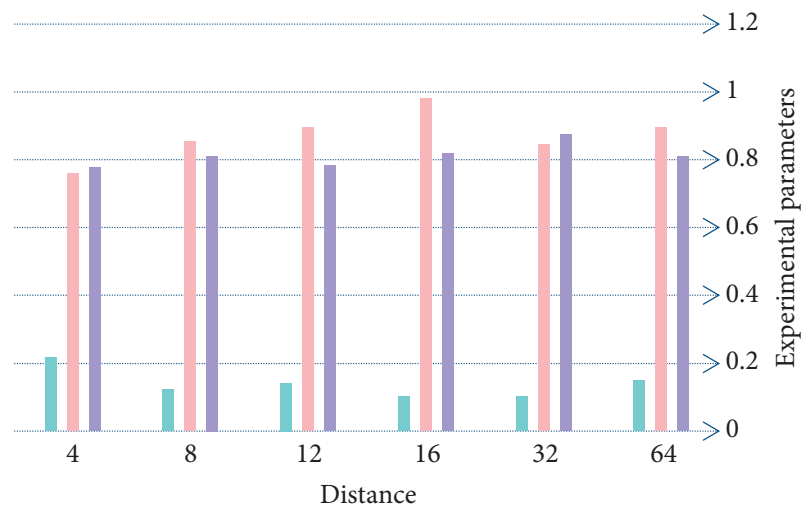

Average error

$\operatorname{Pr}\left(\operatorname{err}<0.3^{\circ}\right)$

FIgURE 2: SOM neural network prediction results of noise-free signal AOA.

TABLE 2: Comparison of AOA errors predicted by SOM neural network and RBF neural network.

\begin{tabular}{lccccccccc}
\hline$x$ & 0 & 5 & 10 & 15 & 20 & 25 & 30 & 35 & 40 \\
\hline BRF & 0.43 & -0.05 & 0.08 & -0.16 & 0.09 & 6.25 & 15.64 & 16.28 & 17.89 \\
SOM & -0.08 & 0.04 & -0.11 & 0.03 & 0.08 & 0.13 & 0.01 & 0.04 & 0.02 \\
\hline
\end{tabular}

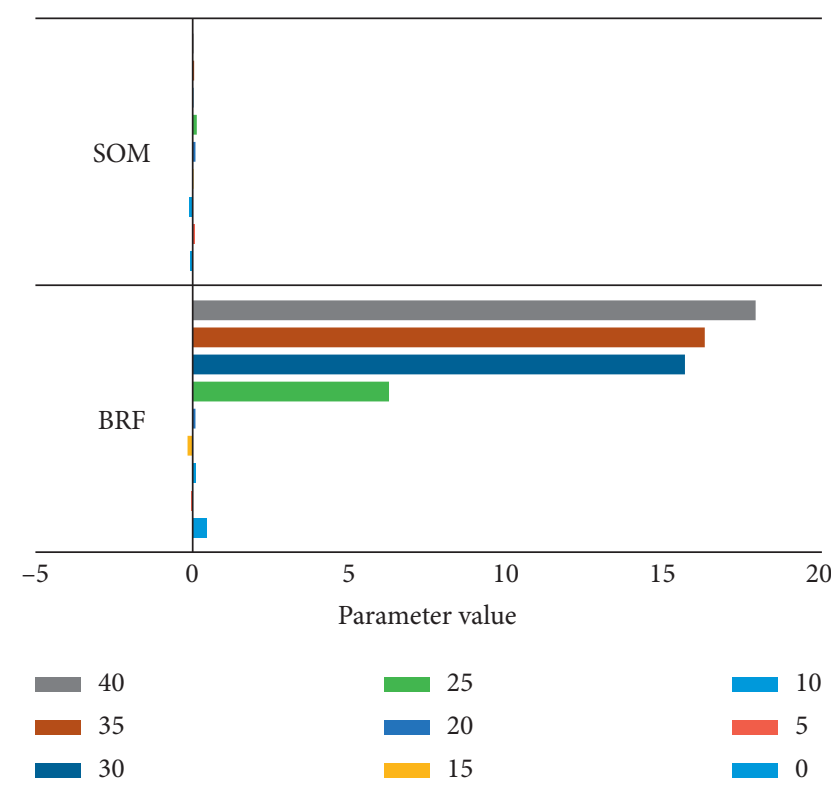

FIgure 3: Comparison of AOA errors predicted by SOM neural network and RBF neural network. 


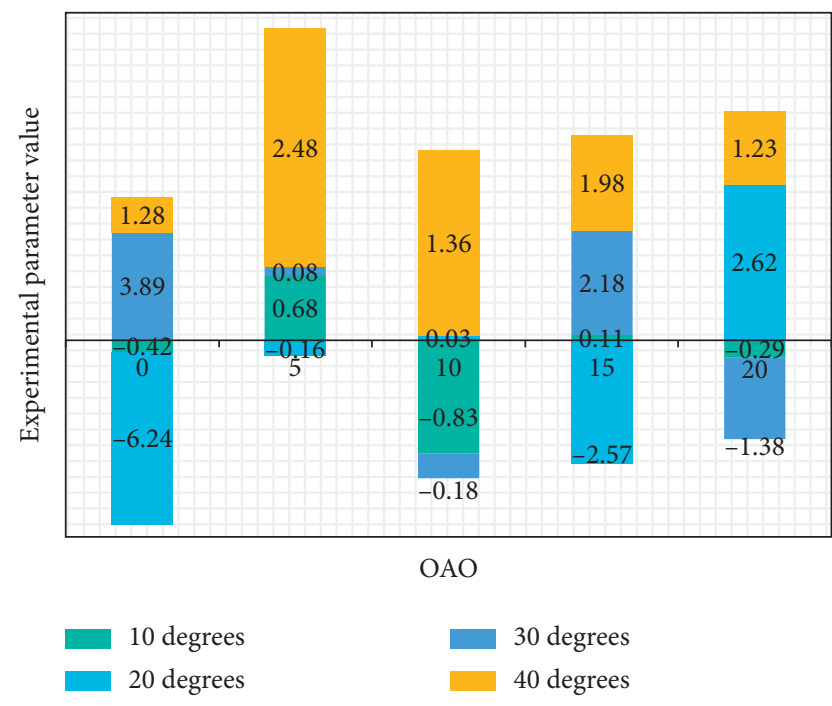

FIGURE 4: SOM neural network predicts AOA resulting in the case of additional Gaussian noise.

4.2. Simulation Experiment with Gaussian Noise. In practical applications, the signal received by the sensor array is often noisy. So here we use the signal containing Gaussian white noise to test the abovementioned SOM neural network, and the test results are shown in Table 3.

It can be seen from Figure 4 that when the signal-tonoise ratio drops from $20 \mathrm{~dB}$ to $1 \mathrm{~dB}$, the absolute error of the AOA prediction is small and the fluctuations are not large; that is, when the signal-to-noise ratio is greater than $1 \mathrm{~dB}$, we establish that the prediction effect of the SOM network does not decrease with the decrease of the signal-to-noise ratio, and it has strong adaptability to noise.

\subsection{Experimental Analysis of Underwater Experiment Results} in the Lake. During the experiment, the hydrophone array was placed at a depth of 3.7 meters below the surface of the water to be fixed. The sound source to be measured is produced by a transducer. Here, the transducer is placed at a depth consistent with the depth of the hydrophone, and the sound wave frequency is $2 \mathrm{KHz}$. The position and direction of the sound source are changed by changing the position of the transducer. When the transducer is at a certain position, the hydrophone array receives the signal, considering the speed of the sound signal in the water and the time difference of the signal after noise reduction, and we can get the DDOA vector. Input the $\mathrm{DDOA}$ vector into the pretrained SOM neural network to get the estimated value of AOA. The estimated results are shown in Table 4.

As shown in Figure 5, the results of the noise-free signal test show that this method is ideal for both near-field signals and far-field signals; the test results for signals containing Gaussian white noise reflect the high prediction accuracy of this method under low signal-to-noise ratios. Further, the applicability of this method in actual experiments can be seen from the results of lake water field experiments.

This paper uses the two-layer SOM neural network proposed earlier and inputs the DDOA vector consistent
TABLE 3: SOM neural network predicts AOA resulting in the case of additional Gaussian noise.

\begin{tabular}{lccccc}
\hline AOA (degrees) & 0 & 5 & 10 & 15 & 20 \\
\hline 10 & -0.42 & 0.68 & -0.83 & 0.11 & -0.29 \\
20 & -6.24 & -0.16 & 0.03 & -2.57 & 2.62 \\
30 & 3.89 & 0.08 & -0.18 & 2.18 & -1.38 \\
40 & 1.28 & 2.48 & 1.36 & 1.98 & 1.23 \\
\hline
\end{tabular}

TABLE 4: AOA prediction results of the experiment in the lake.

\begin{tabular}{lccc}
\hline Actual AOA & Sound distance $(\mathrm{m})$ & Forecast AOA & Absolute error \\
\hline 85 & 900 & 78.5 & 7.4 \\
55 & 500 & 47.3 & 3.8 \\
25 & 200 & 26.2 & 5.2 \\
\hline
\end{tabular}

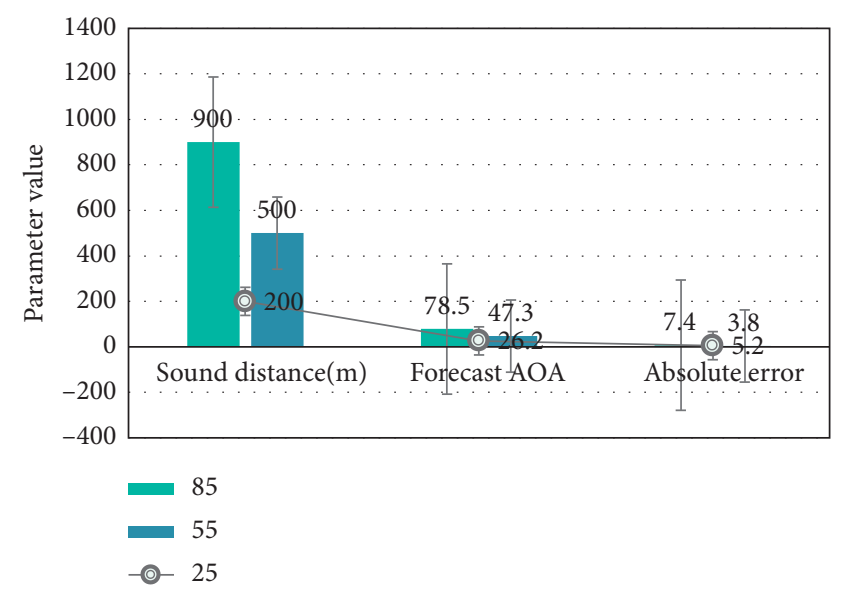

FIgURE 5: AOA prediction results of the experiment in the lake.

with the previous simulation experiment as training data into the network to train the network. Assuming that similar DDOA vectors also correspond to similar signal emission positions, the signal emission positions are also estimated according to the aforementioned three neuron activation situations and principles. The test signal emission points are selected as 20 points in the area where the simulation signal used for training the network is located, and they are evenly distributed along the curve. The test results are shown in Table 5.

The test result is shown in Figure 6, the actual position point number is consistent with the abscissa, and the corresponding network estimated position is marked with a number. It can be seen that, except for a few points, the estimated deviation is small, and the other deviations are still large. Therefore, the hypothesis is not true, and this twolayer SOM neural network is not suitable for the estimation of signal transmission position.

4.4. Comparison and Analysis of Results. The category center of K-means clustering method is generated based on a limited number of iterations, while the category center of genetic clustering is generated by global search. Therefore, in terms of the clustering effect and the robustness of the 
TABLE 5: Using SOM neural network to predict signal position results.

\begin{tabular}{lccccc}
\hline$X$ & 0 & 5 & 10 & 15 & 20 \\
\hline Real & 3.68 & 5.18 & 6.63 & 8.46 & 17.36 \\
Estimation & 4.95 & 6.32 & 7.13 & 10.47 & 15.36 \\
\hline
\end{tabular}

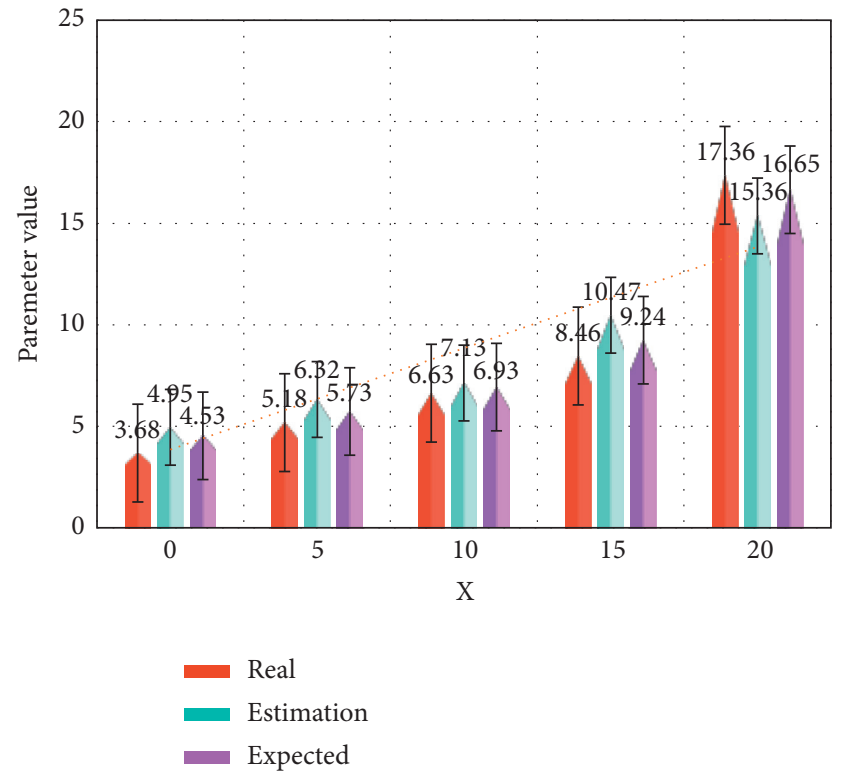

FIGURE 6: Using SOM neural network to predict signal position results.

category center, genetic clustering algorithm is better than kmeans clustering method in these two points. Table 6 lists the ratio of the sum of the interclass distances to the sum of intraclass distances obtained by using these two methods, respectively, that is, the fitness function.

It can be seen from Figure 7 that, compared to the $\mathrm{K}$-means clustering method, the genetic clustering method has obvious advantages in the "cohesion effect" of the cluster center. Especially for RBF networks, the "cohesion effect" of the center often has a greater impact on the performance of the network, so although from the perspective of purely physical or chemical clustering analysis, genetic clustering is not necessarily superior to $\mathrm{K}$-means method, in terms of the effect of RBF network learning, genetic clustering has greater advantages compared to K-means clustering.

\subsection{Influence of the Number of Neuron Nodes on the Prediction} Effect. In order to study the influence of the number of neuron nodes in the network on the prediction effect, simulation experiments were carried out on 6 different neuron node distribution modes. The absolute value of the absolute error of the experimental results is then averaged, as shown in Table 7.

As shown in Figure 8, it can be seen that the network prediction effect using the signal on the circle as the training
TABLE 6: Comparison of genetic clustering and K-means clustering methods.

\begin{tabular}{lcc}
\hline Number of categories & \multicolumn{2}{c}{ Fitness } \\
& $\begin{array}{c}\text { K-means clustering } \\
\text { method }\end{array}$ & $\begin{array}{c}\text { Genetic clustering } \\
\text { method }\end{array}$ \\
\hline 4 & 3.26 & 4.61 \\
5 & 3.52 & 4.78 \\
6 & 3.78 & 4.98 \\
7 & 3.62 & 5.18 \\
8 & 3.29 & 4.72 \\
\hline
\end{tabular}

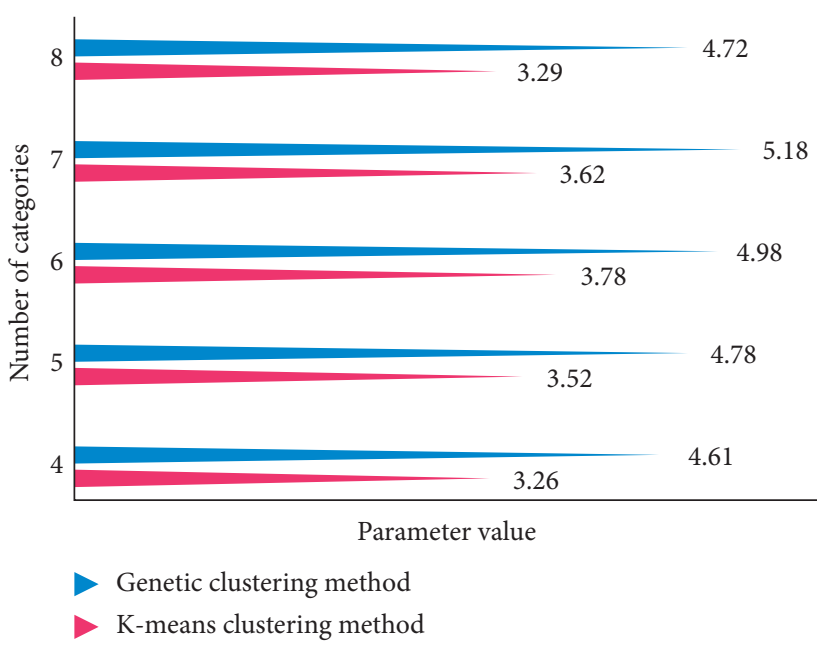

Figure 7: Comparison of genetic clustering and K-means clustering methods.

TABLE 7: Average absolute value of AOA prediction error under different neuron node arrangements.

\begin{tabular}{lcccccc}
\hline $\begin{array}{l}\text { Node } \\
\text { arrangement }\end{array}$ & $20 \times 20$ & $25 \times 25$ & $30 \times 30$ & $35 \times 35$ & $40 \times 40$ & $45 \times 45$ \\
$\begin{array}{l}\text { Rectangular } \\
\text { domain }\end{array}$ & 0.72 & 0.61 & 0.57 & 0.48 & 0.55 & 0.62 \\
Circle & 0.51 & 0.56 & 0.42 & 0.37 & 0.33 & 0.41 \\
\hline
\end{tabular}

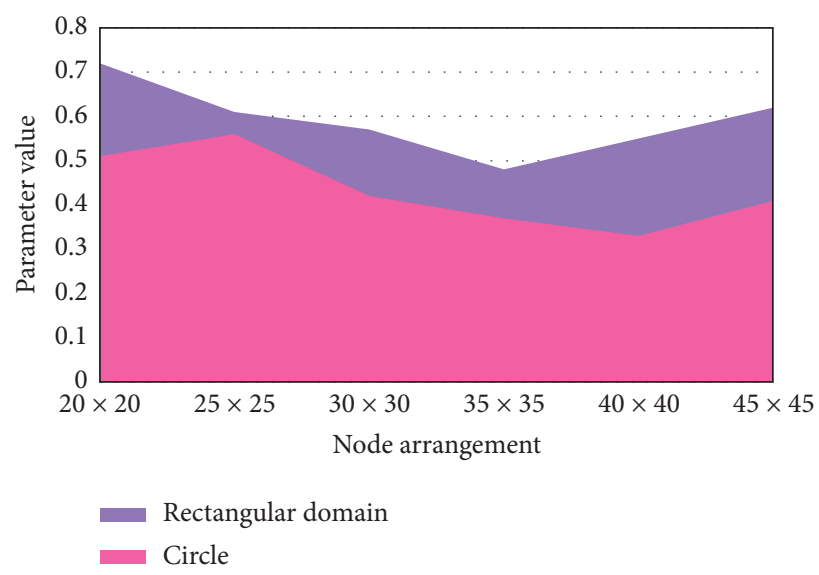

FIgURE 8: Average absolute value of AOA prediction error under different neuron node arrangements. 
data is better than that of the signal training network in the rectangular area. The prediction accuracy of the network with a neuron node distribution of $40 \times 40$ is relatively better than that of other node distributions. This distribution is an $n \times n$ square matrix arrangement, which is slightly smaller in number than the number of training samples. It can also be seen that the prediction effect of the network is not better as the number of neuron nodes increases.

\section{Conclusions}

In this paper, a two-layer SOM neural network is used to study the AOA prediction problem based on DDOA vectors under arbitrary arrays in theory and simulation experiments. This network is equivalent to a classifier, through the classification of DDOA vectors to achieve the classification of AOA values, so as to achieve the purpose of predicting AOA. The established two-layer SOM neural network is further discussed, and the feasible situation of applying the network for prediction is given. First, clarify the features used for prediction and form the input vector, and the predicted object is used as the output of the network.

This method is verified through simulation experiments and actual lake experiments. From the experimental results, it can be seen that the neural network trained in advance through simulation data can detect the direction of arrival of the source signal without noise, Gaussian white noise, and real noise environment, and the angle estimation effect is good. Finally, we further compare the prediction effect of this method with the classic MUSIC algorithm and RBF neural network method. The experimental results show that the performance of this network is excellent and can be considered for practice.

This paper applies SOM neural network to the estimation of the direction of arrival of array signals. It is found through research that the DDOA vector and AOA in the array signal have similar topological distributions. Based on this, the SOM neural network is connected with the topological order to establish a two-layer SOM neural network to estimate the direction of arrival of the array signal. While the method has a theoretical basis, it also shows high estimation accuracy in both simulation experiments and lake water experiments.

\section{Data Availability}

The data that support the findings of this study are available from the corresponding author upon reasonable request.

\section{Conflicts of Interest}

The authors declare that they have no conflicts of interest regarding the publication of the research article.

\section{Acknowledgments}

This work was supported by the National Natural Science Foundation of China under Grant 61703280.

\section{References}

[1] X. Li, Y. Wang, and G. Liu, "Structured medical pathology data hiding information association mining algorithm based on optimized convolutional neural network," IEEE access, vol. 8, no. 1, pp. 1443-1452, 2020.

[2] M. A. A. Mamun, M. A. Hannan, A. Hussain, and H. Basri, "Theoretical model and implementation of a real time intelligent bin status monitoring system using rule based decision algorithms," Expert Systems with Applications, vol. 48, pp. 76-88, 2016.

[3] M. F. Hamza, H. J. Yap, and I. A. Choudhury, "Recent advances on the use of meta-heuristic optimization algorithms to optimize the type-2 fuzzy logic systems in intelligent control," Neural Computing and Applications, vol. 28, no. 5, pp. 1-21, 2015.

[4] B. Tom and S. Alexei, "Conditional random fields for pattern recognition applied to structured data," Algorithms, vol. 8, no. 3, pp. 466-483, 2015.

[5] Y. Chen, W. Zheng, W. Li, and Y. Huang, "The robustness and sustainability of port logistics systems for emergency supplies from overseas," Journal of Advanced Transportation, vol. 2020, Article ID 8868533, 10 pages, 2020.

[6] W. Quan, "Intelligent information processing," Computing in Science \& Engineering, vol. 21, no. 6, pp. 4-5, 2019.

[7] X. Q. Cheng, X. W. Liu, J. H. Li et al., "Data optimization of traffic video vehicle detector based on cloud platform," Jiaotong Yunshu Xitong Gongcheng Yu Xinxi/Journal of Transportation Systems Engineering and Information Technology, vol. 15, no. 2, pp. 76-80, 2015.

[8] S. Wei, Z. Xiaorui, P. Srinivas et al., "A self-adaptive dynamic recognition model for fatigue driving based on multi-source information and two levels of fusion," Sensors, vol. 15, no. 9, pp. 24191-24213, 2015.

[9] M. Niu, S. Sun, J. Wu, and Y. Zhang, "Short-term wind speed hybrid forecasting model based on bias correcting study and its application," Mathematical Problems in Engineering, vol. 2015, no. 10, 13 pages, 2015.

[10] X. Song, X. Li, and W. Zhang, "Key parameters estimation and adaptive warning strategy for rear-end collision of vehicle," Mathematical Problems in Engineering, vol. 2015, no. 20, 20 pages, Article ID 328029, 2015.

[11] M. El-Banna, "A novel approach for classifying imbalance welding data: mahalanobis genetic algorithm (MGA)," International Journal of Advanced Manufacturing Technology, vol. 77, no. 1-4, pp. 407-425, 2015.

[12] J. P. Amezquita-Sanchez and H. Adeli, "Signal processing techniques for vibration-based health monitoring of smart structures," Archives of Computational Methods in Engineering, vol. 23, no. 1, pp. 1-15, 2016.

[13] C. Li, X. An, and R. Li, "A chaos embedded GSA-SVM hybrid system for classification," Neural Computing and Applications, vol. 26, no. 3, pp. 713-721, 2014.

[14] A. Abboud, F. Iutzeler, R. Couillet, M. Debbah, and H. Siguerdidjane, "Distributed production-sharing optimization and application to power grid networks," IEEE Transactions on Signal and Information Processing Over Networks, vol. 2, no. 1, pp. 16-28, 2016.

[15] X. Xu, D. Cao, Y. Zhou et al., "Application of neural network algorithm in fault diagnosis of mechanical intelligence," Mechanical Systems and Signal Processing, vol. 141, no. Jul, pp. 106625.1-106625.13, 2020. 
[16] H. Xiao, B. Biggio, B. Nelson, H. Xiao, C. Eckert, and F. Roli, "Support vector machines under adversarial label contamination," Neurocomputing, vol. 160, no. jul.21, pp. 53-62, 2015.

[17] G. Kan, C. Yao, Q. Li et al., "Improving event-based rainfallrunoff simulation using an ensemble artificial neural network based hybrid data-driven model," Stochastic Environmental Research \& Risk Assessment, vol. 29, no. 5, pp. 1345-1370, 2015.

[18] S. Cuomo, G. De Pietro, R. Farina, A. Galletti, and G. Sannino, "A novel O ( n ) numerical scheme for ECG signal denoising," Procedia Computer Science, vol. 51, no. 1, pp. 775-784, 2015.

[19] H. Guo, G. Dai, J. Fan, Y. Wu, F. Shen, and Y. Hu, "A mobile sensing system for UrbanPM2.5Monitoring with adaptive resolution," Journal of Sensors, vol. 2016, no. 9, 15 pages, Article ID 7901245, 2016.

[20] Q. Liu, J. Liu, R. Sang et al., "Fast neural network training on FPGA using quasi-Newton optimization method," IEEE Transactions on Very Large Scale Integration (VLSI) Systems, vol. 26, no. 99, pp. 1575-1579, 2018.

[21] R. M. S. F. Almeida and V. P. De Freitas, "An insulation thickness optimization methodology for school buildings rehabilitation combining artificial neural networks and life cycle cost," Journal of Civil Engineering and Management, vol. 22, no. 7, pp. 915-923, 2016.

[22] A. S. Soma, T. Kubota, and H. Mizuno, "Optimization of causative factors using logistic regression and artificial neural network models for landslide susceptibility assessment in Ujung Loe Watershed, South Sulawesi Indonesia," Journal of Mountain Science, vol. 16, no. 2, pp. 383-401, 2019.

[23] S. C. Miao, J. H. Yang, X. H. Wang et al., "Blade pattern optimization of the hydraulic turbine based onneural network and genetic algorithm," Hangkong Dongli Xuebao/Journal of Aerospace Power, vol. 30, no. 8, pp. 1918-1925, 2015.

[24] Z. Junsheng, Y. Gu, and Z. Feng, "Optimization of processing parameters of power spinning for bushing based on neural network and genetic algorithms," Journal of Bjing Institute of Technology, vol. 28, no. 3, pp. 228-238, 2019.

[25] N. Melzi, L. Khaouane, S. Hanini, M. Laidi, Y. Ammi, and H. Zentou, "Optimization methodology of artificial neural network models for predicting molecular diffusion coefficients for polar and non-polar binary gases," Journal of Applied Mechanics and Technical Physics, vol. 61, no. 2, pp. 207-216, 2020.

[26] N. Chen, B. Rong, X. Zhang, and M. Kadoch, "Scalable and flexible massive MIMO precoding for 5G H-cran," IEEE Wireless Communications, vol. 24, no. 1, pp. 46-52, 2017.

[27] R. Noorossana, A. Zadbood, F. Zandi et al., "An interactive artificial neural networks approach to multiresponse optimization," International Journal of Advanced Manufacturing Technology, vol. 76, no. 5-8, pp. 765-777, 2015.

[28] D. Sánchez, P. Melin, and O. Castillo, "Optimization of modular granular neural networks using a firefly algorithm for human recognition," Engineering Applications of Artificial Intelligence, vol. 64, no. sep, pp. 172-186, 2017. 